BULL. AUSTRAL. MATH. SOC.

$20-04,20015$

VOL. 9 (1973), 433-438.

\title{
Computer results on Burnside groups
}

\section{I.D. Macdonald}

Power and commutator structure tables are obtained for a number of factor groups of Burnside groups.

This is an interim report on results which are presently being obtained by the computer methods described in [5]. Developments of those methods and applications to the calculation of multiplicators have already been described in [6]. This report deals with developments in another direction, applications to finite p-groups of given exponent and particularly to certain factor groups of Burnside groups. It seems likely from the results to be described here that much new material could be obtained, given sufficient computing power, and it has been decided to publish a report now while the calculations are in the process of being transferred from the ICL 4130 at the University of Stirling to the Edinburgh Regional Computing Centre IBM 370/155.

As usual $B(m, n)$ will denote the Burnside group of exponent $m$ on $n$ generators, and in this note $B(m, n)_{c}$ will denote the group $G / \Upsilon_{c+1}(G)$ where $G=B(m, n)$; for other notation we refer to [5].

Our results concern the power and commutator structure of various groups $B(m, n)_{c}$, notably $B(5,2)_{6}$, of order $5^{14}$, and $B(4,3)_{6}$, of order $2^{63}$. They are not given in detail here, but the author will furnish them on request. Hall has explained in [1] and [2] the interest attached to finding the order of $B(4,3)$, for instance, and though we are not yet in a position to find this order we do construct a factor group of order $2^{63}$. The other results are similar in nature.

Received 6 July 1973. 
The chief alteration to the computer programme described in [5] has been the calling of a procedure EXPON which has superseded the use of defining relations. At each stage where relations would normally be used (see [5]), it is arranged by means of EXPON that $x^{m}=1$ for each element $x$ of the group under investigation, $m$ being as above the given exponent.

But of course it would be quite impossible to verify directly that every element of the group had a correct order, since we are interested in quite large groups. Fortunately it suffices to verify that $x^{m}=1$ for $x$ varying in a reasonably small subset of the group, if we take advantage of the fact that we are working in a nilpotent $p$-group. The precise subset depends, as we shall see, on judicious handling of special cases.

Let us start with a simple and therefore clear instance. If we consider $B(5,2)$ and in particular its factor group $B(5,2)_{6}$ then the following lemma is relevant.

LEMMA I'. If $G=\operatorname{gp}\{a, b\}$ is a finite 5-group of class 6 , if

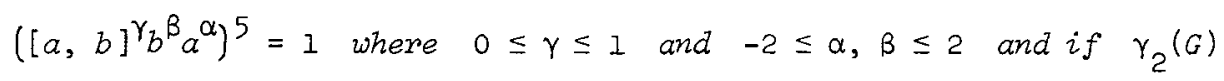
has exponent 5 , then $G$ has exponent 5 . //

It is no doubt easy to prove this simple result by hand calculation, but we wish here to exemplify invocation of machine aid, in the following way. In [5] a programme called BASIC was mentioned (and even claimed to be "of relatively little use in investigations of groups"); it merely defines basic commutators and does straightforward collections in terms of them. Now in the ordinary way we have basic commutators of weight 1 - let us call them [1], ..., [N] in notation similar to that used by the machine and the remaining basic commutators are cormutators in $\{[1], \ldots,[N]\}$. However there is no need to assign weight $I$ to the "undefined" commutators $[1], \ldots,[N]$, they may be given arbitrary weights and (definitions being inspired as always by what is needed in the collection process) the machine can be told to define "basic" commutators in $\{[1], \ldots,[N]\}$ and to assign appropriate weights to them.

Next suppose $N=3$ and that $W T[1]=1, W T[2]=2, W T[3]=2$. Basic commutators in $\{[1],[2],[3]\}$ are defined and collection of

$$
([2][1])^{5},([3][1])^{5},([3][2][1])^{5}
$$


respectively up to and including weight 6 is carred out by means of the amended BASIC. Examination of the results shows that

$$
([3][2][1])^{5}=([3][1])^{5}([2][1])^{5}
$$

provided that the exponents of all basic commutators are reduced modulo 5. This we state in group-theoretical terms as:

LEMMA 1. Let $F$ be the free nilpotent group of class 6 on a set $S$ and let $x, y_{1}, y_{2}$ be commutators of weight $1,2,2$ respectively in elements of $S$. Then, in the group generated by $s_{1}=\left\{x, y_{1}, y_{2}\right\}$,

$$
\left(y_{2} y_{1} x\right)^{5}=\left(y_{2} x\right)^{5}\left(y_{1} x\right)^{5}
$$

modulo the normal closure of the subgroup generated by the fifth powers of al2 basic commutators in elements of $S_{1}$.

Lemma $I^{\prime}$ follows from Lemma 1 . During the machine construction we use EXPON to make $(y x)^{5}=1$ where $y$ runs through basic commutators of weight at least 2 and $x$ through a set of coset representatives of $\gamma_{2}(G)$ in $G$; it is quite easy to see that we need take $y$ of weight 2 only. In the constructed group $G$ we find that $\gamma_{2}(G)$, a 5-group of class 3 , is generated by elements of order 5 , and is therefore regular. So $\gamma_{2}(G)$ has exponent 5 , and Lemma $I^{\prime}$ applies.

The group has order $5^{14}$ and the ranks of the elementary abelian groups $\gamma_{i}(G) / \gamma_{i++}(G)$ are

$$
2,1,2,3,2,4
$$

for $1 \leq i \leq 6$; these figures agree with those given by Kostrikin [3]. We remark that the only fifth powers used by the machine in place of relations were $a^{5}$ and $\left(b a^{\alpha}\right)^{5}$ for $\alpha=-2,-1,0,1,2$. The working indicates that $B(5,2)_{5}$ has multiplicator of rank at least 8 , but we have not made a systematic determination of multiplicators.

Now we turn to 2 -groups and in particular to $B(4,3)_{6}$. Since the techniques here are entirely similar in principle to those used for 
$B(5,2)_{6}$ we may state analogues of Lemmas 1 and 1 ' without comment:

LEMMA 2. Let $F$ be the free nilpotent group of class 6 on a set $S$ and let $x, y_{1}, y_{2}$ be commutators of weight $1,2,2$ respectively in elements of $S$. Then, in the group generated by $s_{1}=\left\{x, y_{1}, y_{2}\right\}$,

$$
\left(y_{2} y_{1} x\right)^{4}=\left(y_{2} x\right)^{4}\left(y_{1} x\right)^{4}
$$

modulo the normal closure of the subgroup generated by the fourth powers of all basic commitators in elements of $S_{1}$ and by the element

$$
\left[x, y_{1} ; x, y_{2}\right]\left[y_{2}, x, x, y_{1}\right]\left[y_{1}, x, x, y_{2}\right] .
$$

LEMMA 2'. Suppose that $G=\operatorname{gp}\{a, b, c\}$ is a finite 2-group of class 6 satisfying the following conditions:

(i) $(y x)^{4}=1$ where $y$ mus through $\{1,[a, b],[a, c],[b, c]\}$ and $x$ runs through $\left\{c^{\gamma} b^{\beta}{ }^{\alpha}: 0 \leq \alpha, \beta, \gamma \leq 3\right\}$;

(ii) $\gamma_{4}(G)$ has exponent 2 ;

(iii) $\left[x, y_{1} ; x, y_{2}\right]\left[y_{2}, x, x, y_{1}\right]\left[y_{1}, x, x, y_{2}\right]=1$ where $x$ rons through elements of weight $I$ and $y_{1}, y_{2}$ through elements of weight 2 .

Then $G$ has exponent 4 . //

Verification of ( $i i)$ of Lemma $2^{\prime}$ is done by inspection of the computer output. The ranks of the first six lower central factors of $B(4,3)$ are

$$
3,3,8,17,21,8 \text {. }
$$

One reason for investigating $B(4,3)_{6}$ was that it might lead to a finite 2-group $G$, not of exponent 4 , but having $x^{4}=1$ for every element not in $\gamma_{2}(G)$. As it happens such a group $G$ does not emerge in this case, for scrutiny of the computer output shows that the fourth-power relations used by the machine to define $B(4,3)_{6}$ were all of the form $x^{4}$ for $x$ outside the commutator subgroup. (In fact the programme was written so that such relations are those chosen first.) 
Another approach to the groups $B(4, n)$ is to consider factor groups in which some of the $n$ generators have order 2 . This has been introduced by Leech [4]. Our programme soon showed that if two generators of $B(4,3)$ are taken to have order 2 then a group of class 5 and order $2^{19}$ results, while tables were produced for $G / \gamma_{7}(G)$ where $G$ is the factor group of $B(4,3)$ - with $\{a, b, c\}$ as free generators - got by taking $e^{2}=1$. The order of $G / \Upsilon_{7}(G)$ is $2^{36}$, and the ranks of the lower central factors are

$$
3,3,6,11,9,2 \text {; }
$$

some hand calculation gives the further result that $\gamma_{7}(G) / \gamma_{8}(G)$ has order at most 2 .

Other groups investigated to date are $B(8,2)$ and $B(9,2)$. Since these are very large and no results of great interest have been found we shall not give any details. In the case of $B(8,2)_{6}$ we merely report that the order is $2^{35}$, and that $\gamma_{6} / \gamma_{7}$ has exponent 2 .

As for exponent 9 , even groups generated by two elements of order 3 lead to depressingly large results. Thus in the free case the ranks of the first few lower central factors were found to be

$$
2,1,2,2,2,2,4,5,8 \text {. }
$$

The only relations used by the machine were $a^{3}=b^{3}=1$, $(b a)^{9}=\left(b a^{-1}\right)^{9}=1$. (We note that the standard wreath product of $B(3,3)$ with $B(3,3)$ which is a group of exponent 9 generated by 6 elements of order 3 , has class at least 45 by Theorem 3.5 of [7].) AIl this tends to suggest that there are extremely large groups of exponent 9 generated by $\{a, b\}$ where $a^{3}=b^{3}=1$.

On the other hand if the further relation $(b a)^{3}=1$ is added then $a$ group of order $3^{5}$ and class 4 resulted. This is the largest nilpotent 3-group of exponent 9 satisfying the 3 relations mentioned. 


\section{References}

[1] Marshall Hall, Jr, "Discrete variable problems", Survey of numerical analysis, 518-542 (McGraw-Hill, New York, San Francisco, Toronto, London; 1962).

[2] Marshall Hall, Jr and D.E. Knuth, "Groups of exponent 4 ", Notices Amer. Math. Soc. 11 (1964), 120-121.

[3] А.Н. Ностринин [А.І. Kostrikin], "Решенис ослабленной проблегы Бернсайда для поназателя 5 " [Solution of the restricted Burnside problem for exponent 5 ], Izv. Akad. Nauk SSSR Ser. Mat. 19 (1955), 233-244.

[4] John Leech, "Coset enumeration on digital computers", Proc. Combridge Philos. Soc. 59 (1963), 257-267.

[5] I.D. Macdonald, "A computer application to finite p-groups", J. Austral. Math. Soc. (to appear).

[6] I.D. Macdonald and J.W. Wamsley, "On the multiplicator of finite p-groups", (in preparation).

[7] Teresa Scruton, "Bounds for the class of nilpotent wreath products", Proc. Cambridge Phizos. Soc. 62 (1966), 165-169.

[8] C.R.B. Wright, "On the nilpotency class of a group of exponent four", Pacific J. Math. 11 (1961), 387-394.

Department of Mathematics,

University of Stirling,

stirling,

Scotland. 